\title{
The Directed and Rubinov Subdifferentials of Quasidifferentiable Functions, Part I: Definition and Examples
}

\author{
Robert Baier $^{\mathrm{a}, 1, *}$, Elza Farkhi ${ }^{\mathrm{b}, 1}$, Vera Roshchina ${ }^{\mathrm{c}}$ \\ ${ }^{a}$ Chair of Applied Mathematics, University of Bayreuth, 95440 Bayreuth, Germany \\ ${ }^{b}$ School of Mathematical Sciences, Sackler Faculty of Exact Sciences, \\ Tel Aviv University, 69978 Tel Aviv, Israel \\ ${ }^{c}$ CIMA-UE, FCT - Ciência 2008, University of Evora, 7000-671 Évora, Portugal
}

\begin{abstract}
We extend the definition of the directed subdifferential, originally introduced in [R. Baier, E. Farkhi: The directed subdifferential of DC functions, in: A. Leizarowitz, B. S. Mordukhovich, I. Shafrir, A. J. Zaslavski (Eds.), Nonlinear Analysis and Optimization II: Optimization. A Conference in Celebration of Alex Ioffe's 70th and Simeon Reich's 60th Birthdays, June 18-24, 2008, Haifa, Israel, in: AMS Contemp. Mathem. 513, AMS and Bar-Ilan University, 2010, pp. 27-43], for differences of convex functions (DC) to the wider class of quasidifferentiable functions. Such generalization efficiently captures differential properties of a wide class of functions including amenable and lower/upper- $\mathrm{C}^{k}$ functions. While preserving the most important properties of the quasidifferential, such as exact calculus rules, the directed subdifferential lacks the major drawbacks of quasidifferential: non-uniqueness and "inflation in size" of the two convex sets representing the quasidifferential after applying calculus rules. The Rubinov subdifferential is defined as the visualization of the directed subdifferential.
\end{abstract}

Keywords: subdifferentials, quasidifferentiable functions, differences of sets, directed sets, directed subdifferential, amenable and lower- ${ }^{k}$ functions 2010 MSC: 49J52, 26B25, 90C26

\footnotetext{
*corresponding author

Email addresses: robert.baier@uni-bayreuth.de (Robert Baier), elza@post.tau.ac.il (Elza Farkhi), vera.roshchina@gmail.com (Vera Roshchina)

${ }^{1}$ partially supported by The Hermann Minkowski Center for Geometry at Tel Aviv University, Tel Aviv, Israel

Preprint submitted to Nonlinear Analysis

August 8, 2011
} 


\section{Introduction}

The directed subdifferential is first introduced in [4] for a DC (difference of convex) function $f=g-h$ with $g, h$ convex, as the difference of the convex subdifferentials of $g$ and $h$, embedded in the Banach space of directed sets [2]. The construction of the directed sets enables not only to compute differences (and other operations) of embedded convex sets, but also to visualize them [3]. The difference of convex compact sets embedded in this space provides an interesting alternative to the non-constructive representation of differences of convex compacts by pairs of sets (see e.g. [22, 7, 20]) The Rubinov subdifferential, defined here as the visualization of the directed subdifferential, is a compact, generally non-convex set in $\mathbb{R}^{n}$.

In [4] the question whether the directed subdifferential may be extended beyond the class of DC functions, was posed. Here we propose such an extension to the class of quasidifferentiable (QD) functions. The reader may find examples with the visualization of the directed subdifferential for the special case of DC functions in $[4,6]$. Here, we focus our attention mainly on theoretical investigation of this extension to QD functions. The second part of this work [5] deals with calculus rules of the directed subdifferential, connections to other subdifferentials and with optimality conditions.

The class of QD functions (see e.g. [7, 17, 20]) attracted our attention for several reasons.

First, being essentially wider than the class of DC functions, it preserves the property of the latter that the directional derivative of a function in this class is the difference of two sublinear functions, that is, the difference of the support functions of two convex compacts. Thus, it is natural to define the directed subdifferential of a QD function as the difference of these two compacts embedded in the space of directed sets. These two compacts (up to a negation) compose also the quasidifferential of the function [7], which is an equivalence class of pairs of convex sets (see $[22,20]$ ).

Second, as we show here, the class of QD functions is so rich that besides the DC functions (see [7, Subsec. III.2.1, 2. and Theorem 2.1]), it also contains the $\mathrm{C}^{1}$ functions, the amenable functions and lower/upper- $\mathrm{C}^{k}$ functions $(k \geq 1)$ which are introduced and discussed in [23], [24, Definitions 10.23 and 10.29] and [17, Sec. 3.2, remarks before Corollary 3.76]. Since this large class of QD functions is closed under linear combinations and finite minimum and maximum [7, Chap. III, Theorem 2.1], all functions obtained by these operations between functions of all mentioned types remain in the class of QD 
functions. Therefore, the space of QD functions is not only a vector space, but also a vector lattice (Riesz space), if the supremum of two functions is defined as their usual maximum.

Third, besides being wider than the class of DC functions, QD functions allow a relatively easy construction of the quasidifferential, mostly thanks to the fact that it is easier to see the DC structure of a positively homogeneous mapping (i.e. the directional derivative) than of a general DC function. Recall that constructing a DC representation of even a $\mathrm{C}^{2}$ function is not a trivial problem. We often come across optimization problems which deal with simple quasidifferentiable functions which involve compositions of a rather limited set of functions - pointwise minima and maxima of smooth functions, norms, etc. The calculus of quasidifferentials for such QD functions is well developed, and offers clear and exact rules for computing quasidifferentials. Our construction allows to benefit of these calculus rules, and to avoid disadvantages of the quasidifferential, as the non-uniqueness in the representation by pairs of sets and the "inflation in size" for the representing sets. For example, if calculus rules are used to compute one representation of the quasidifferential of the function $f(x)=r\|x\|-r\|x\|$ at zero, we would get a pair of balls of radius $r$, not two zero singletons, as one would expect that from $f(x) \equiv 0$ (for details, see Example 3.3 in the second part of this paper [5]). At the same time, the directed subdifferential of this function is zero no matter how it was computed. The non-uniqueness and "inflation in size" stimulated the investigation of minimal pairs, see [20].

Calculus rules and other properties of the directed subdifferential are postponed to the second part of this work [5].

The paper is organized as follows. In Section 2 we introduce some notation and remind a few basic definitions related to convex analysis and differences of convex compacts. In Section 3 we introduce the notion of quasidifferentiable functions and remind some examples of such functions. We then explain the idea behind directed sets, define the directed subdifferential of a QD function and show the correctness of such definition in Section 4. Finally, in Section 5 the important subclasses of QD functions are discussed: the amenable and lower- $\mathrm{C}^{k}$ functions.

\section{Preliminaries}

Throughout the paper we try to adhere to the standard notation: we use the Euclidean norm $\|\cdot\|$, and denote by $S_{n-1}$ the unit sphere in $\mathbb{R}^{n}$. 
For any sets $A, B \subset \mathbb{R}^{n}$ the relevant linear operations are defined as follows

$$
\begin{aligned}
& A+B:=\{a+b \mid a \in A, b \in B\} \quad \text { (Minkowski addition), } \\
& \lambda \cdot A:=\{\lambda \cdot a \mid a \in A\} \quad \text { (scalar multiplication for } \lambda \in \mathbb{R} \text { ), } \\
& \ominus A:=\{-a \mid a \in A\} \quad \text { (pointwise negation of } A \text { ), } \\
& A \ominus B \quad:=A+(\ominus B)=\{a-b \mid a \in A, b \in B\} \quad \text { (algebraic difference). }
\end{aligned}
$$

The cone $\mathcal{C}\left(\mathbb{R}^{n}\right)$ of nonempty convex and compact subsets of $\mathbb{R}^{n}$ is of a special importance in our framework. The support function $\delta^{*}(\cdot, A): \mathbb{R}^{n} \rightarrow \mathbb{R}$ for a set $A \in \mathcal{C}\left(\mathbb{R}^{n}\right)$ is defined as $\delta^{*}(l, A):=\max _{a \in A}\langle l, a\rangle$ for each direction $l \in \mathbb{R}^{n}$. It is Lipschitz-continuous and fulfills

$$
\delta^{*}(l, \lambda A+\mu B)=\lambda \delta^{*}(l, A)+\mu \delta^{*}(l, B), \quad(\lambda, \mu \geq 0) .
$$

The Hausdorff distance between two sets in $\mathcal{C}\left(\mathbb{R}^{n}\right)$ is expressed via the support function

$$
\mathrm{d}_{\mathrm{H}}(A, B)=\max _{l \in S_{n-1}}\left|\delta^{*}(l, A)-\delta^{*}(l, B)\right| .
$$

The support face (set of supporting points) for the direction $l \in S_{n-1}$ is

$$
Y(l, A):=\left\{y(l, A) \in A \mid\langle l, y(l, A)\rangle=\delta^{*}(l, A)\right\} .
$$

There are several ways to define differences of convex sets. Apart from the Hadwiger-Pontryagin or star-shaped difference [9, 21]

$$
A^{*} B:=\left\{x \in \mathbb{R}^{n} \mid x+B \subset A\right\}
$$

(which has got the desirable property $A^{*} A=\{0\}$, but is empty in many important cases) we also use Demyanov difference [7, 25]

$$
\begin{aligned}
A-B:=\overline{\operatorname{co}}\{Y(l, A)-Y(l, B) \mid & l \in S_{n-1}, \\
& Y(l, A) \text { and } Y(l, B) \text { are singletons }\} .
\end{aligned}
$$

The norm of this difference, i.e. the maximal norm of one of its elements, yields the Demyanov metric

$$
\partial_{D}(A, B)=\|A-B\|
$$

(see [8, Sec. 4]) which is stronger than the Hausdorff metric. 


\section{Quasidifferentiable functions}

In a nutshell, quasidifferentiable functions [7] develop the idea of DC functions further, allowing to deal with a local convex-concave structure of the directional derivative instead of worrying about the DC representation of the whole function. Apart for relative ease in construction of a quasidifferential (compared to establish a rather nontrivial DC representation), QD functions allow for simple calculus rules in the form of equalities.

\subsection{Definition and some basic properties}

Recall that a function $f: \mathbb{R}^{n} \rightarrow \mathbb{R}$ is (Dini) directionally differentiable at a point $x \in \mathbb{R}^{n}$ if the limit

$$
f^{\prime}(x ; l):=\lim _{t \downarrow 0} \frac{f(x+t l)-f(x)}{t}
$$

exists and is finite for all $l \in \mathbb{R}^{n}$. Quasidifferentiable functions are the ones for which the directional derivative is a specific DC function as a function of the direction. We give a formal definition now.

Definition 3.1. The function $f: \mathbb{R}^{n} \rightarrow \mathbb{R}$ is quasidifferentiable (QD) at the point $x \in \mathbb{R}^{n}$, if it is directionally differentiable at $x$ in any direction and there exist two convex compacts $\underline{\partial} f(x), \bar{\partial} f(x) \in \mathcal{C}\left(\mathbb{R}^{n}\right)$ with

$$
f^{\prime}(x ; l)=\max _{u \in \underline{\partial} f(x)}\langle l, u\rangle+\min _{v \in \bar{\partial} f(x)}\langle l, v\rangle=\delta^{*}(l, \underline{\partial} f(x))-\delta^{*}(l, \ominus \bar{\partial} f(x)) .
$$

We call the set $\underline{\partial} f(x)$ a quasisubdifferential and $\bar{\partial} f(x)$ a quasisuperdifferential of $f$ at $x$.

A peculiar feature of a quasidifferential is its non-uniqueness, that is, the same function can have an infinite number of different representations (4).

Remark 3.2. The pair of compact sets $[\underline{\partial} f(x), \bar{\partial} f(x)]$ is not unique, since every pair equivalent to it in the sense of [20, 22], i.e. every pair $[A, B]$ satisfying $\underline{\partial} f(x)+B=\bar{\partial} f(x)+A$, may replace the pair $[\underline{\partial} f(x), \bar{\partial} f(x)]$ in the above definition. Thus, the quasidifferential $\mathcal{D} f(x)$ is the equivalence class generated by $[\underline{\partial} f(x), \bar{\partial} f(x)]$, see for details [7, Sec. III.2]. We denote this shortly as $\mathcal{D} f(x)=[\underline{\partial} f(x), \bar{\partial} f(x)]$.

This seemingly unpleasant property has, however, motivated interesting results on equivalence, minimality and reduction of pairs of convex sets mostly due to D. Pallaschke and R. Urbański (e.g. see [19, 20]). 
As every linear mapping is clearly DC, this yields quasidifferentiability of differentiable functions straight away.

Remark 3.3. Every Fréchet (and even Gâteaux) differentiable function is QD, see [7, Subsec. III.2.1, 1.], since its directional derivative may be expressed by $f^{\prime}(x ; l)=\nabla f(x) \cdot l$ which is the support function of the singleton set $\underline{\partial} f(x)=\left\{\nabla f(x)^{\top}\right\}$, and $\bar{\partial} f(x)=\{0\}$.

Also, every $D C$ function $f(x)=g(x)-h(x)$ is $Q D$ with $\underline{\partial} f(x)=\partial g(x)$ and $\bar{\partial} f(x)=\ominus \partial h(x)$ (see [7, Subsec. III.2.1, 2. and Chap. III, Theorem 2.1] and [20, Sec. 10.2.1]).

Recall that the directional derivative of a $\mathrm{QD}$ function is itself QD.

Lemma 3.4. Let $f: \mathbb{R}^{n} \rightarrow \mathbb{R}$ be $Q D$. Then its directional derivative is $Q D$ with

$$
\begin{aligned}
{\left[f^{\prime}(x ; \cdot)\right]^{\prime}(l ; \eta) } & =\delta^{*}(\eta, Y(l, \underline{\partial} f(x)))-\delta^{*}(\eta, \ominus Y(-l, \bar{\partial} f(x))), \\
\mathcal{D}\left[f^{\prime}(x ; \cdot)\right](l) & =[Y(l, \underline{\partial} f(x)), Y(-l, \bar{\partial} f(x))] .
\end{aligned}
$$

Proof. By [12, Chap. VI, Example 3.1, (3.1)] the directional derivative of the support function of a set $C \in \mathcal{C}\left(\mathbb{R}^{n}\right)$ exists and satisfies

$$
\left[\delta^{*}(\cdot, C)\right]^{\prime}(l ; \eta)=\delta^{*}(\eta, Y(l, C)) .
$$

Hence,

$$
\begin{aligned}
{\left[f^{\prime}(x ; \cdot)\right]^{\prime}(l ; \eta) } & =\left[\delta^{*}(\cdot, \underline{\partial} f(x))\right]^{\prime}(l ; \eta)-\left[\delta^{*}(\cdot, \ominus \bar{\partial} f(x))\right]^{\prime}(l ; \eta) \\
& =\delta^{*}(\eta, Y(l, \underline{\partial} f(x)))-\delta^{*}(\eta, \ominus Y(-l, \bar{\partial} f(x)))
\end{aligned}
$$

and the formula for the quasidifferential for the directional derivative follows.

\subsection{Examples}

To give a better idea of what QD functions are (and what they are not) we provide a few illustrative examples, which are already well-known from the literature. Instructive examples for quasidifferentiable functions may be found in [26, 27] and [7, Sec. III.2 and III.4]. More illustrative examples with functions having bounded/unbounded variation (which happens to be an important property for establishing quasidifferentiability) are also available in [10, Chap. Five, Sec. 17-18]. 
The following example of a QD function which is not DC, appears in [18, p. 13 and Fig. 2] and [11, Example 1], citing A. Shapiro [26]. It also shows that a uniform limit of DC functions need not be DC. In this example, the limit function is QD.

Example 3.5. The function $f:[-1,2] \rightarrow \mathbb{R}$ defined by

$$
f(x)=\inf _{k \in \mathbb{N}}\left|x-\frac{1}{k}\right|=\lim _{M \rightarrow \infty} f_{M}(x) \quad \text { with } \quad f_{M}(x)=\min _{1 \leq k \leq M}\left|x-\frac{1}{k}\right|
$$

is $Q D$, but not DC. It is plotted in Fig. 1 .
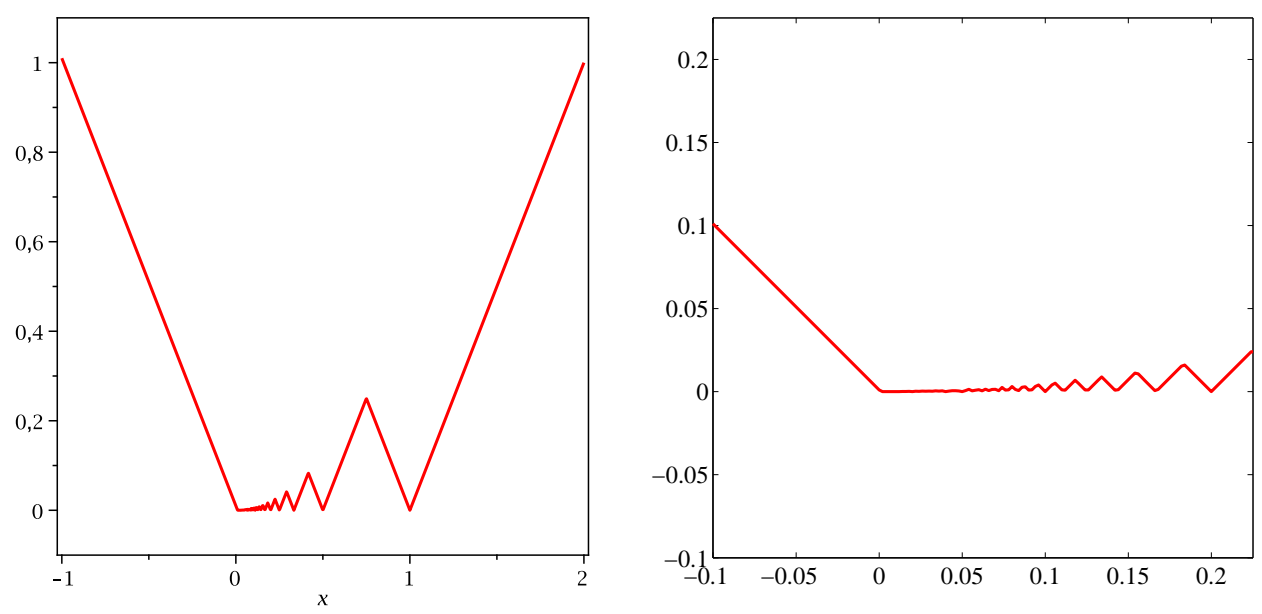

Figure 1: QD function $f_{M}$ with $M=100$ of Example 3.5 (right: zoom)

It is not difficult to observe that the function $f$ is differentiable everywhere except for the points $\left\{t_{k}\right\}_{1}^{\infty},\left\{m_{k}\right\}_{1}^{\infty}$ and 0 , where $t_{k}=\frac{1}{k}$ and $m_{k}=$ $\frac{1}{2}\left(\frac{1}{k}+\frac{1}{k+1}\right)$ for all $k=1, \ldots, \infty$.

To see that the function $f$ is $Q D$, compute the quasidifferential in these cases:

(i) $x>1$ or $x \in\left(t^{k+1}, m^{k}\right): f^{\prime}(x ; \ell)=\ell, \underline{\partial} f(x)=\{1\}, \bar{\partial} f(x)=\{0\}$

(ii) $x<0$ or $x \in\left(m^{k}, t^{k}\right): f^{\prime}(x ; \ell)=-\ell, \underline{\partial} f(x)=\{-1\}$, $\bar{\partial} f(x)=\{0\}$

(iii) $x=m^{k}: f^{\prime}(x ; \ell)=-|\ell|, \underline{\partial} f(x)=\{0\}, \bar{\partial} f(x)=[-1,1]$

(iv) $x=t^{k}: f^{\prime}(x ; \ell)=|\ell|, \underline{\partial} f(x)=[-1,1], \bar{\partial} f(x)=\{0\}$ 
(v) $x=0: f^{\prime}(x ; \ell)=\max \{0,-\ell\}, \underline{\partial} f(x)=[-1,0], \bar{\partial} f(x)=\{0\}$

Thus, for any $x$ one may determine $\underline{\partial} f(x)$ and $\bar{\partial} f(x)$.

To show that the Lipschitz function $f$ is not DC it is sufficient to show that its derivative $f^{\prime}$ is not of bounded variation, since otherwise $f^{\prime}$ would be the difference of two monotone functions, which is a contradiction. As one can see in the plots in Fig. 2-3, the values of the derivative $f^{\prime}$ switch infinitely many times from -1 to 1 at the points $\frac{1}{k}, \frac{1}{2}\left(\frac{1}{k}+\frac{1}{k+1}\right), k \in \mathbb{N}$. The variation of $f^{\prime}$ is not bounded, as the (bounded) variations of $f_{M}^{\prime}$ tend to infinity, if $M \rightarrow \infty$. Hence, $f$ is not $D C$.
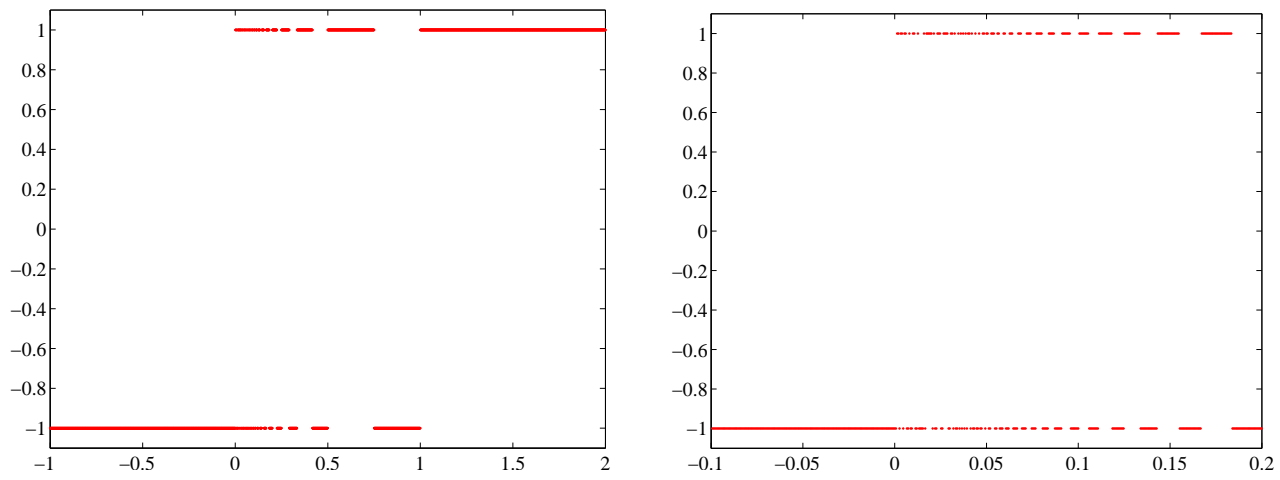

Figure 2: Derivative of QD function of Example 3.5 (right: zoom)
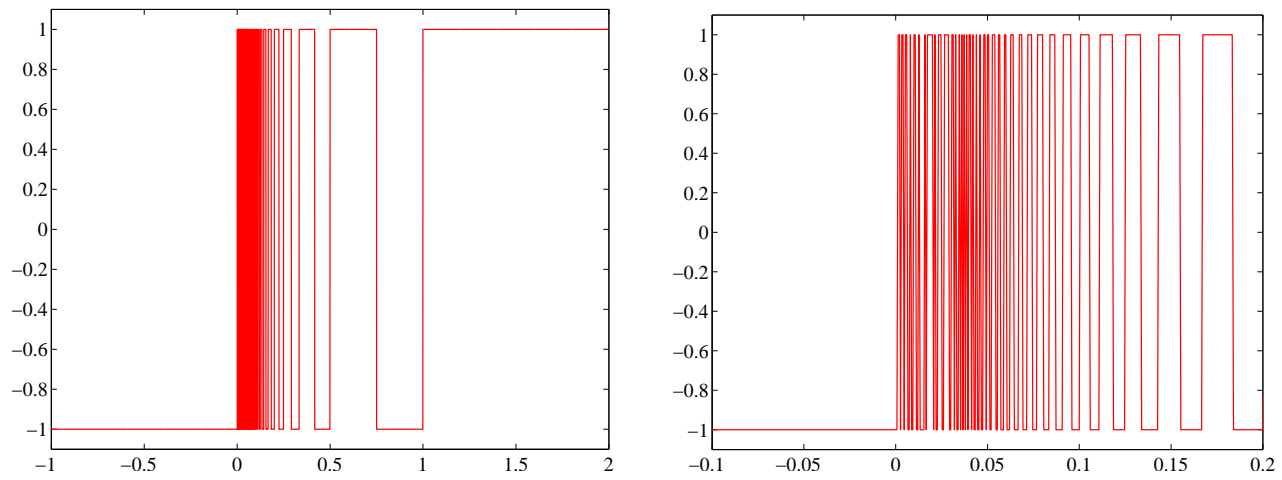

Figure 3: Discontinuity jumps of the derivative of $f$, Example 3.5 (right: zoom)

We conclude from the above example that the set of DC functions is not closed. Indeed, the sequence $\left\{f_{M}\right\}_{M}$ of DC functions considered above (see [7, Sec. III.1, Lemma 1.1]) tends w.r.t. supremum norm to $f$ which is not DC. 
A small variant of the previous example shows that a uniform limit of $\mathrm{DC} / \mathrm{QD}$ functions need not be QD either. In this example, the limit function is only continuous, but is not quasidifferentiable at $x=0$.

Example 3.6. Consider the functions

$$
f_{M}(x)= \begin{cases}\min _{k=1, \ldots, M}|k x-1| & \text { if } x \geq \frac{1}{M}, \quad(M \in \mathbb{N}) \\ 0 & \text { else }\end{cases}
$$

and the limit function $f:[-1,2] \rightarrow \mathbb{R}$ with

$$
f(x)= \begin{cases}\inf _{k \in \mathbb{N}}|k x-1| & \text { if } x \geq 0 \\ 0, & \text { else. }\end{cases}
$$

The function and its derivative are plotted in Fig. 4 and 5.
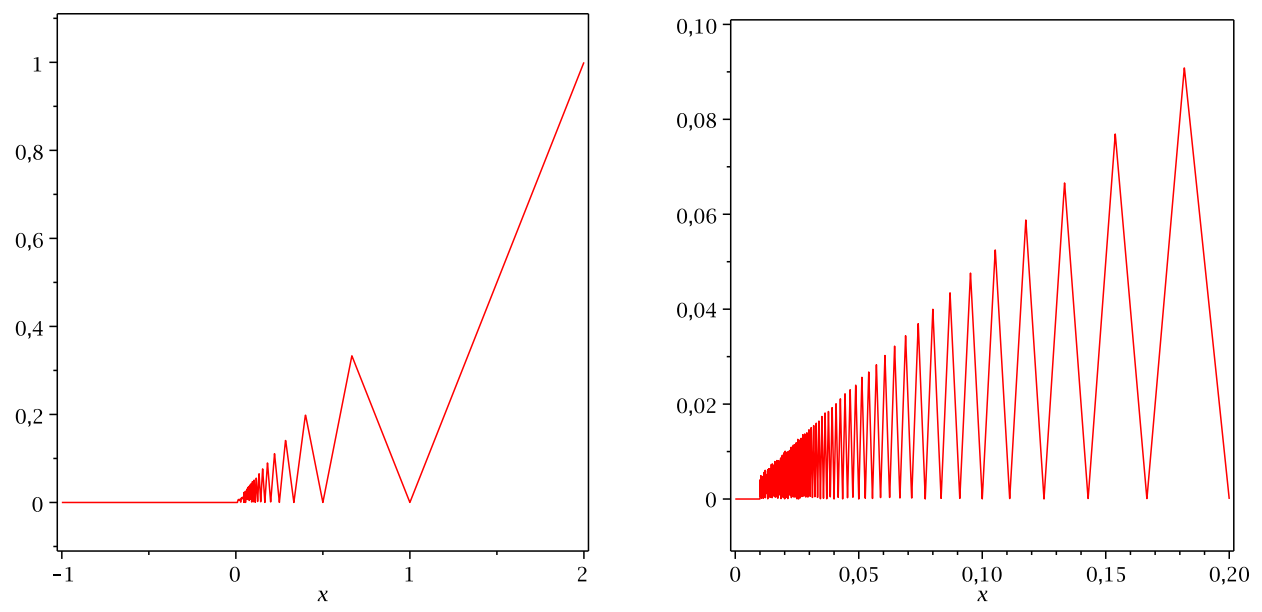

Figure 4: QD function $f_{M}$ with $M=100$ of Example 3.6 (right: zoom)

It is clear that $f(x)=f_{M}(x)$ for $x \geq \frac{1}{M}$.

Set $t^{k}:=\frac{1}{k}$ and $m^{k}:=\frac{2}{2 k+1}$ for $k \in \mathbb{N}$. Then $t^{k}$ are the zeros (local minima) and $m^{k}$ are the local maxima, with $f\left(m^{k}\right)=\frac{1}{2 k+1}$. It follows that $f$ and $f^{\prime}$ have unbounded variation.

To see that $f$ is not $Q D$ at $x=0$, we show that $f$ is not quasidifferentiable for $x=0$, namely we show that $f$ is not directionally differentiable in $x=0$ for direction +1 . 

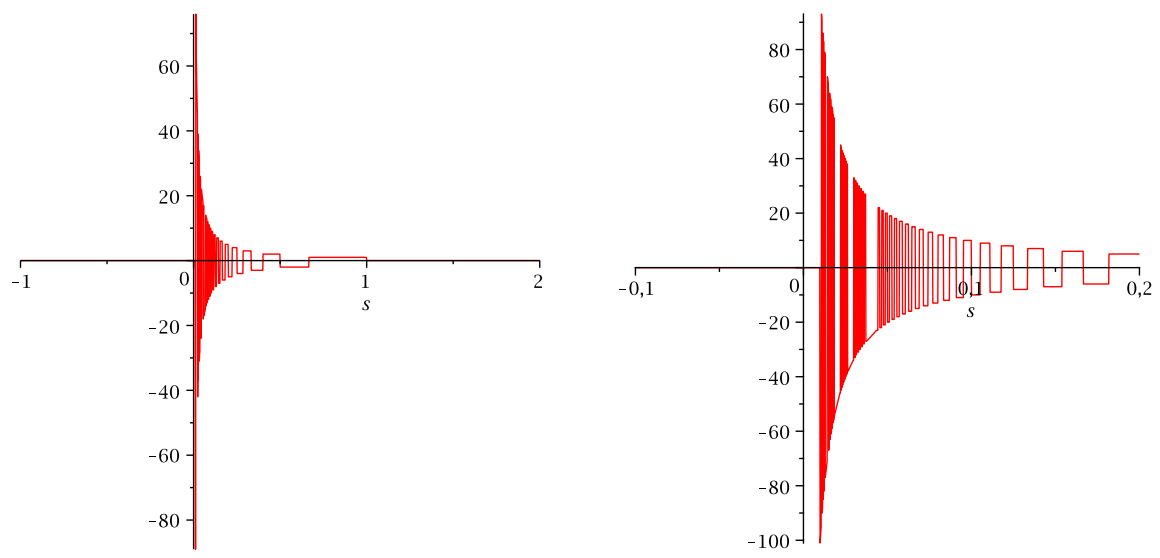

Figure 5: The derivative of $f_{M}$ with $M=100$ of Example 3.6 (right: zoom)

Indeed, for the sequence $\left\{t^{k}\right\}_{k}$ :

$$
\lim _{k \rightarrow \infty} \frac{f\left(t^{k}\right)-f(0)}{t^{k}}=\lim _{k \rightarrow \infty} \frac{0-0}{t^{k}}=0
$$

and for the sequence $\left\{m^{k}\right\}_{k}$ :

$$
\lim _{k \rightarrow \infty} \frac{f\left(m^{k}\right)-f(0)}{m^{k}}=\lim _{k \rightarrow \infty} \frac{\frac{1}{2 k+1}-0}{\frac{2}{2 k+1}}=\frac{1}{2} .
$$

It is well-known that every $\mathrm{C}^{2}$ function is DC (see e.g. [11], [14, Proposition 2.3], [13, Corollary 4.1]).

As we saw, every $\mathrm{C}^{1}$ function is $\mathrm{QD}$, but it may not be $\mathrm{DC}$ as the following example shows.

Example 3.7. Consider the function from Example 3.6, i.e.

$$
g(x)= \begin{cases}\inf _{k \in \mathbb{N}}|k x-1|, & \text { if } x \geq 0 \\ 0, & \text { else }\end{cases}
$$

and its integral

$$
f(x)=\int_{0}^{x} g(t) d t
$$

The function, its first and second derivative are plotted in Fig. 6-8. 

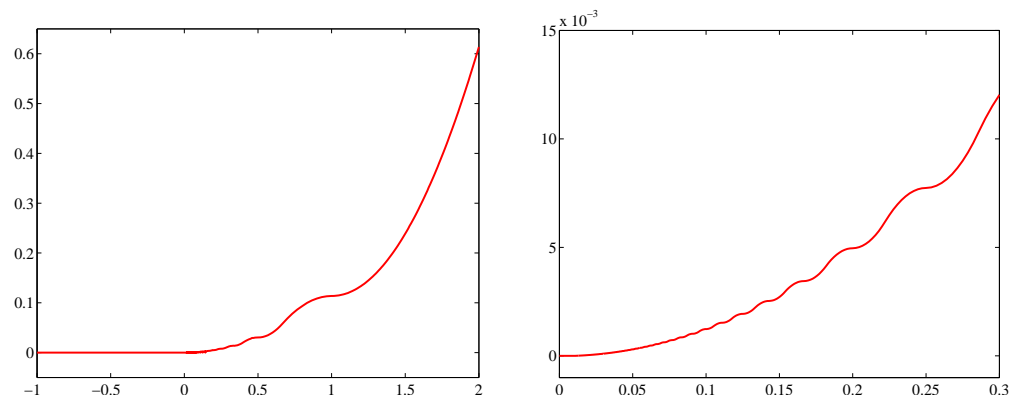

Figure 6: QD function $f_{M}$ with $M=100$ of Example 3.7 (right: zoom)
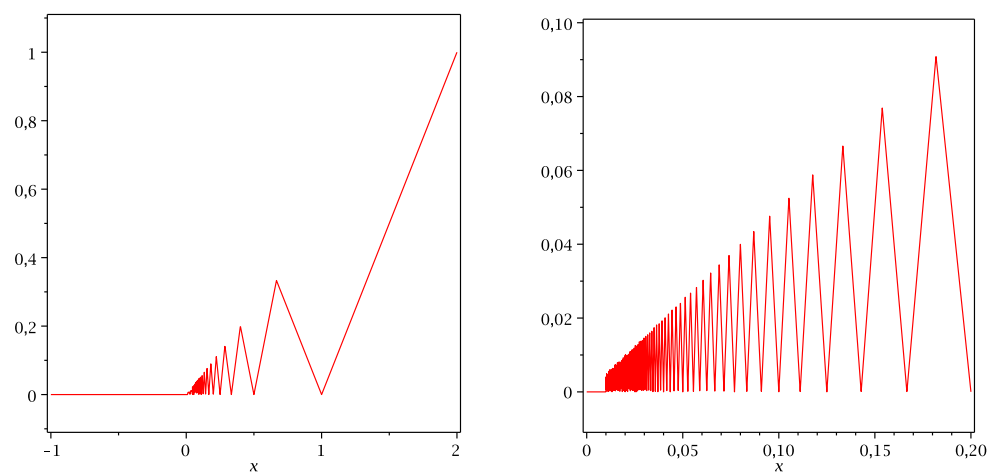

Figure 7: Derivative of QD function $f_{M}$ with $M=100$ of Example 3.7 (right: zoom)
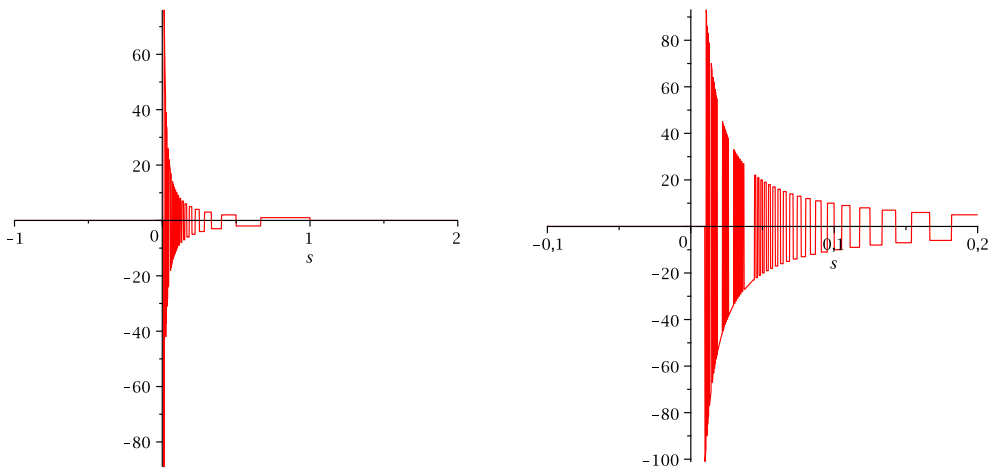

Figure 8: Second derivative of function $f_{M}$ with $M=100$ of Example 3.7 (right: zoom) 
Note that $f$ is $C^{1}([-1,2])$, but not $C^{2}([-1,2])$, since otherwise $f^{\prime}=g$ should be Lipschitz. This contradicts to the unbounded variation of $g$ in Example 3.6, hence, $f$ cannot be $D C$. It is, however, $Q D$, since $f$ is differentiable in $[-1,2]$.

\section{Directed sets and the directed subdifferential}

\subsection{Directed sets}

Since the directed subdifferential belongs to the space of directed sets in $\mathbb{R}^{n}$, we first recall some definitions and facts related to this space $[2,3]$.

A directed set is a kind of "oriented", non-necessarily convex subset of $\mathbb{R}^{n}$. We define directed sets recursively with respect to the dimension $n$ and parametrized by the normal vectors of their lower-dimensional boundary. Thus we are able to construct a Banach space in which subtraction of embedded convex compact sets is well-defined. Using the parametrization of convex compacts via their support functions, we define a directed set as a pair of two mappings that associates to each unit direction a lower-dimensional directed set (corresponding to a supporting face) and a scalar (corresponding to the value of the support function in this direction). This is the base of the recursive constructions.

The definition of directed sets is inductive in the dimension $n \geq 1$. For $n=1$ they are called directed intervals. Our definition of directed intervals is equivalent to the generalized and directed intervals $([15,16])$.

Definition 4.1. A directed interval $\vec{A}$ is a point in $\mathbb{R}^{2}$ or, equivalently, a function $a_{1}:=\{-1,1\} \rightarrow \mathbb{R}$, i.e.

$$
\vec{A}=\left(a_{1}(l)\right)_{l= \pm 1}=\left(a_{1}(-1), a_{1}(1)\right) \in \mathbb{R}^{2} .
$$

$\mathcal{D}(\mathbb{R})$ denotes the set of all directed intervals.

Denote $\overrightarrow{[\alpha, \beta]}:=(-\alpha, \beta)$, where $\alpha=-a_{1}(-1), \beta=a_{1}(1)$.

Linear combinations of directed intervals are calculated as linear combinations of vectors:

$$
\lambda \overrightarrow{\left[\alpha_{1}, \beta_{1}\right]}+\mu \overrightarrow{\left[\alpha_{2}, \beta_{2}\right]}=\overrightarrow{\left[\lambda \alpha_{1}+\mu \alpha_{2}, \lambda \beta_{1}+\mu \beta_{2}\right]}, \quad \lambda, \mu \in \mathbb{R} .
$$

We visualize a directed interval $\overrightarrow{[\alpha, \beta]}$ attaching to each of its end points the corresponding unit normal vector, $l_{1}=-1$ to the point $\alpha$ and $l_{2}=1$ to $\beta$. The directed interval is proper, if $\alpha \leq \beta$ (then the normals are pointing outwards) and improper otherwise (with normals pointing inwards). Some proper and improper intervals are visualized in Example 4.2. 
Example 4.2. One proper and one improper interval, obtained by subtraction of directed intervals are shown here:

$$
\begin{aligned}
& \overrightarrow{[-3,5]}-\overrightarrow{[-1,2]}=\overrightarrow{[-2,3]} \text { and } \overrightarrow{[-1,2]}-\overrightarrow{[-3,5]}=\overrightarrow{[2,-3]}
\end{aligned}
$$

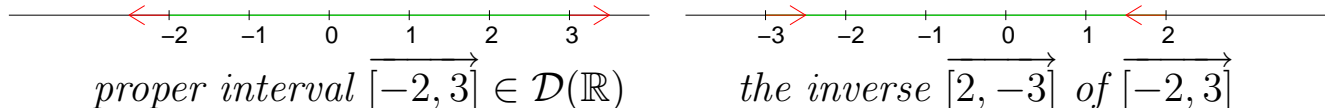

Another improper interval as well as the embedded convex set $\{1\}$ are visualized below:

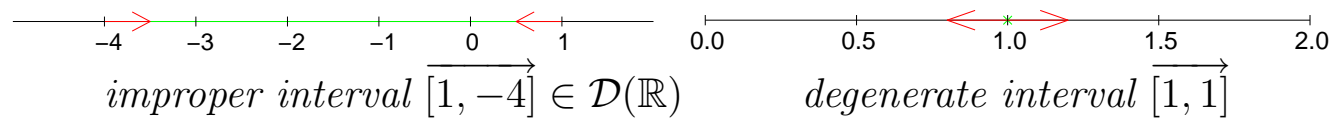

The linear normed space $\mathcal{D}\left(\mathbb{R}^{n}\right)$ of the directed sets in $\mathbb{R}^{n}$ is constructed inductively motivated by representing nonempty convex compact set $A$ by its $(n-1)$-dimensional supporting face and the value of the support function for each direction $l \in S_{n-1}$.

Definition 4.3. $\vec{A}$ is called a directed set

(i) in $\mathbb{R}$, if it is a directed interval. Its norm is $\|\vec{A}\|_{1}=\max _{l= \pm 1}\left|a_{1}(l)\right|$.

(ii) in $\mathbb{R}^{n}, n \geq 2$, if there exist a continuous function $a_{n}: S_{n-1} \rightarrow \mathbb{R}$ and a uniformly bounded map having lower-dimensional directed sets as images $\overrightarrow{A_{n-1}}: S_{n-1} \rightarrow \mathcal{D}\left(\mathbb{R}^{n-1}\right)$ with respect to $\|\cdot\|_{n-1}$.

Then, we denote $\vec{A}=\left(\overrightarrow{A_{n-1}(l)}, a_{n}(l)\right)_{l \in S_{n-1}}$ and define its norm as

$$
\|\vec{A}\|:=\|\vec{A}\|_{n}:=\max \left\{\sup _{l \in S_{n-1}}\left\|\overrightarrow{A_{n-1}(l)}\right\|_{n-1}, \max _{l \in S_{n-1}}\left|a_{n}(l)\right|\right\} .
$$

The set of all directed sets in $\mathbb{R}^{n}$ is denoted by $\mathcal{D}\left(\mathbb{R}^{n}\right)$.

The linear operations are defined recursively on the two components of the directed sets $\vec{A}=\left(\overrightarrow{A_{n-1}(l)}, a_{n}(l)\right)_{l \in S_{n-1}}, \vec{B}=\left(\overrightarrow{B_{n-1}(l)}, b_{n}(l)\right)_{l \in S_{n-1}}$ :

$$
\begin{aligned}
\vec{A}+\vec{B} & :=\left(\overrightarrow{A_{n-1}(l)}+\overrightarrow{B_{n-1}(l)}, a_{n}(l)+b_{n}(l)\right)_{l \in S_{n-1}}, \\
\lambda \cdot \vec{A} & :=\left(\lambda \cdot \overrightarrow{A_{n-1}(l)}, \lambda \cdot a_{n}(l)\right)_{l \in S_{n-1}} \\
\vec{A}-\vec{B} & :=\vec{A}+(-\vec{B})=(\lambda \in \mathbb{R}), \quad\left(\overrightarrow{A_{n-1}(l)}-\overrightarrow{B_{n-1}(l)}, a_{n}(l)-b_{n}(l)\right)_{l \in S_{n-1}}
\end{aligned}
$$


It is proved in [2] that $\left(\mathcal{D}\left(\mathbb{R}^{n}\right),+, \cdot\right)$ is a Banach space, a vector lattice (Riesz space), and for $n=1$ it is a Banach lattice.

The embedding $J_{n}: \mathcal{C}\left(\mathbb{R}^{n}\right) \rightarrow \mathcal{D}\left(\mathbb{R}^{n}\right)$ which determines for every set $A \in$ $\mathcal{C}\left(\mathbb{R}^{n}\right)$ its embedded image $\vec{A} \in \mathcal{D}\left(\mathbb{R}^{n}\right)$ is defined as:

(i) For $n=1, \overrightarrow{[a, b]}=J_{1}([a, b]):=(-a, b)$.

(ii) For $n \geq 2, \vec{A}=J_{n}(A):=\left(J_{n-1}\left(P_{n-1, l}(Y(l, A))\right), \delta^{*}(l, A)\right)_{l \in S_{n-1}}$, where $P_{n-1, l}(x):=\pi_{n-1, n} R_{n, l}(x)$ and $\pi_{n-1, n} \in \mathbb{R}^{(n-1) \times n}$ is the natural projection, $R_{n, l}$ is a fixed rotation for every $l \in S_{n-1}$ satisfying

$$
R_{n, l}(l)=e^{n}, \quad R_{n, l}\left(\operatorname{span}\{l\}^{\perp}\right)=\operatorname{span}\left\{e^{1}, e^{2}, \ldots, e^{n-1}\right\} .
$$

The linear image of a directed set $\vec{U} \in \mathcal{D}\left(\mathbb{R}^{m}\right)$, being a limit of the sequence $\left(J_{m}\left(A_{k}\right)-J_{m}\left(B_{k}\right)\right)_{k}$ with $A_{k}, B_{k} \in \mathcal{C}\left(\mathbb{R}^{m}\right)$, under a linear mapping $M \in \mathbb{R}^{n \times m}$ is defined as in [1, Sec. 3]:

$$
\begin{aligned}
M C & :=\{M c: c \in C\} \\
M \vec{U} & :=\lim _{k \rightarrow \infty}\left(J_{n}\left(M A_{k}\right)-J_{n}\left(M B_{k}\right)\right)
\end{aligned}
$$

The well-definedness and the linearity property is shown in [1, Lemmas 3.1 and 3.2].

The visualization for a directed set in $\mathcal{D}\left(\mathbb{R}^{n}\right)$, which is a compact, generally non-convex set in $\mathbb{R}^{n}$, consists of three parts: the convex, the concave and the mixed-type parts. We recall their definitions.

Definition 4.4. Let $\vec{A} \in \mathcal{D}\left(\mathbb{R}^{n}\right)$. The convex (positive) part $P_{n}(\vec{A})$ and the concave (negative) part $N_{n}(\vec{A})$ of $\vec{A}$ are defined by:

$$
\begin{aligned}
& P_{n}(\vec{A}):=\left\{x \in \mathbb{R}^{n} \mid \text { for every } l \in S_{n-1}:\langle l, x\rangle \leq a_{n}(l)\right\}, \\
& N_{n}(\vec{A}):=\left\{x \in \mathbb{R}^{n} \mid \text { for every } l \in S_{n-1}:\langle l, x\rangle \geq a_{n}(l)\right\} .
\end{aligned}
$$

The mixed-type part $M_{n}(\vec{A})$ is defined recursively and collects all points (being reprojected in $\mathbb{R}^{n}$ ) from the visualization of the component with the lower-dimensional directed sets which are not elements of the convex or concave part.

$$
\begin{array}{ll}
M_{1}(\vec{A}):=\emptyset, \quad V_{1}(\vec{A}):=P_{1}(\vec{A}) \cup N_{1}(\vec{A}) & (n=1), \\
M_{n}(\vec{A}):=\bigcup_{l \in S_{n-1}}\left\{x \in Q_{n, l}\left(V_{n-1}\left(\overrightarrow{A_{n-1}(l)}\right)\right) \mid x \notin P_{n}(\vec{A}) \cup N_{n}(\vec{A})\right\} & (n \geq 2) .
\end{array}
$$


The visualization $V_{n}: \mathcal{D}\left(\mathbb{R}^{n}\right) \Rightarrow \mathbb{R}^{n}$ is the union of the three parts

$$
V_{n}(\vec{A}):=P_{n}(\vec{A}) \cup N_{n}(\vec{A}) \cup M_{n}(\vec{A}) \quad(n \geq 2)
$$

with the reprojection $Q_{n, l}(y):=R_{n, l}^{-1} \pi_{n-1, n}^{\top} y+a_{n}(l) l, y \in \mathbb{R}^{n-1}$. The boundary mapping $B_{n}: \mathcal{D}\left(\mathbb{R}^{n}\right) \Rightarrow \mathbb{R}^{n}$ is defined as

$$
B_{n}(\vec{A}):=\partial P_{n}(\vec{A}) \cup \partial N_{n}(\vec{A}) \cup M_{n}(\vec{A}) .
$$

Let us note that the visualization of an embedded convex compact set $J_{n}(A)$ consists only of a positive part which is the set $A$ itself, and the visualization of the negation $-J_{n}(A)$ has only a negative part which is the set $\ominus A$, see $[3$, Proposition 3.8].

Proposition 4.5. Consider $C \in \mathcal{C}\left(\mathbb{R}^{n}\right)$, the embedded directed set $\vec{A}=$ $J_{n}(C)$ and its inverse $\vec{B}=-J_{n}(C)$.

Then,

$$
\begin{aligned}
& V_{n}(\vec{A})=P_{n}(\vec{A})=C, \\
& V_{n}(\vec{B})=N_{n}(\vec{B})=\ominus C .
\end{aligned}
$$

Except the degenerate case when $V_{n}(\vec{A})$ consists of a single point, at least one of the convex and the concave parts of $A$ is empty. It may happen that both of them are empty and the visualization consists only of the mixed-type part. In the one-dimensional case the mixed-type part is empty, and in the non-degenerate case exactly one of $P_{1}(\overrightarrow{[a, b]})$ and $N_{1}(\overrightarrow{[a, b]})$ is empty.

The visualization of the difference of directed sets is strongly related to other differences. The following equalities are known for $\vec{A} \in \mathcal{D}\left(\mathbb{R}^{n}\right)$, cf. [3]:

$$
P_{n}(-\vec{A})=\ominus N_{n}(\vec{A}), \quad N_{n}(-\vec{A})=\ominus P_{n}(\vec{A}), \quad V_{n}(-\vec{A})=\ominus V_{n}(\vec{A}) .
$$

Example 4.6. Let $A=\left\{(x, y) \in \mathbb{R}^{2} \mid y \geq 0, x^{2}+y^{2} \leq 1\right\}$. The visualizations of $\vec{A}=J_{2}(A)$ and $-\vec{A}$ are shown in Fig. 9. The visualization of the inverse comprises the pointwise negations of the boundary points, keeping the corresponding normal directions $l$.

Note that if the set $A$ is symmetric with respect the origin, then the visualizations of $\vec{A}$ and $-\vec{A}$ coincide, and the only way to distinguish between them is to add the normal vectors to the visualization. 

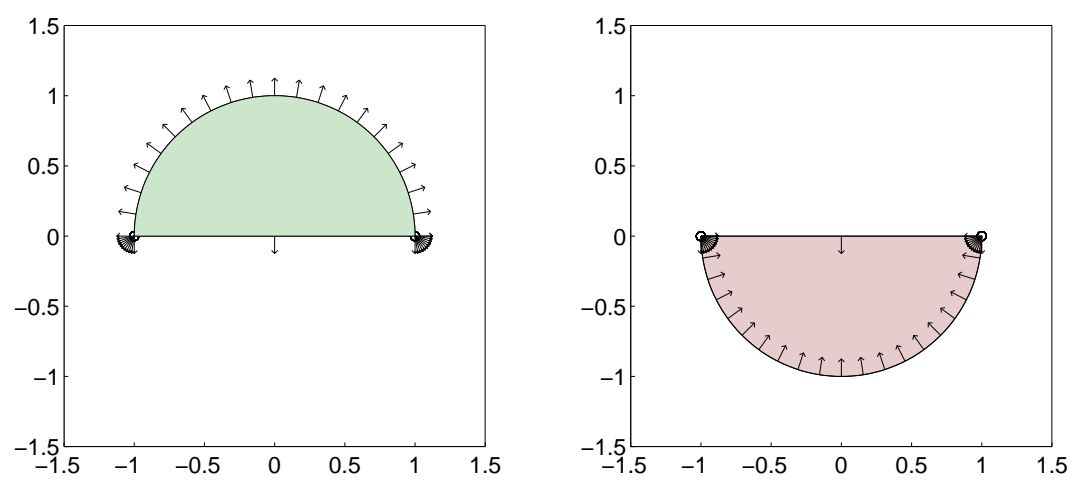

Figure 9: $\vec{A}$ resp. $-\vec{A}$ for $\vec{A}=J_{2}(A), A=\left\{(x, y) \in \mathbb{R}^{2} \mid y \geq 0, x^{2}+y^{2} \leq 1\right\}$

Furthermore, if $A, B \in \mathcal{C}\left(\mathbb{R}^{n}\right)$ and $\vec{A}=J_{n}(A), \vec{B}=J_{n}(B)$, then

$$
\begin{gathered}
P_{n}(\vec{A}-\vec{B})=A * B, \quad N_{n}(\vec{A}-\vec{B})=\ominus(B \stackrel{*}{*}), \\
\overline{\operatorname{co}} B_{n}(\vec{A}-\vec{B})=A-B, \\
\bigcup_{l \in S_{n-1}}\{y(l, A)-y(l, B) \mid Y(l, A), Y(l, B) \text { are singletons }\} \subset B_{n}(\vec{A}-\vec{B}) .
\end{gathered}
$$

The following example is a rather curious demonstration of visualizations of a difference of two embedded convex sets. Just changing sizes of these sets makes visualizations completely different. More details can be found in $[4$, Example 5.7].

Example 4.7. Let $A=[-1,1] \times[-1,1], B_{r}=\left\{x \in \mathbb{R}^{2} \mid\|x\| \leq r\right\}$. The corresponding visualization of the difference of embeddings

$$
V_{2}\left(J_{2}(A)-J_{2}\left(B_{r}\right)\right)
$$

with different values of $r$ are plotted in Fig. 10 (see [4, Example 5.7] for detailed explanation).

The arrows in Fig. 10 indicate outer normals to the directed "supporting faces". The positive part in the left picture of Fig. 10 is a convex set. It is colored in light green and only outer normals are attached to its boundary. The non-convex part belongs to the mixed-type part. Similarly for the right picture in Fig. 10. The light red convex subset is the negative part and only has inner normals attached to its boundary. The positive and negative part in the middle picture are empty and the Rubinov subdifferential consists only of the mixed-type part. 


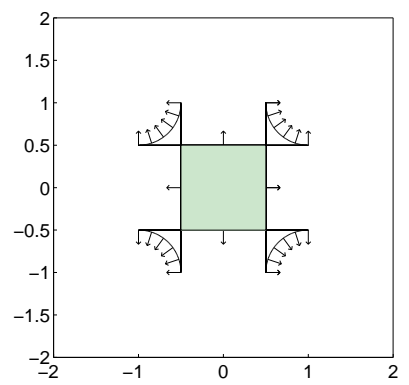

(a)

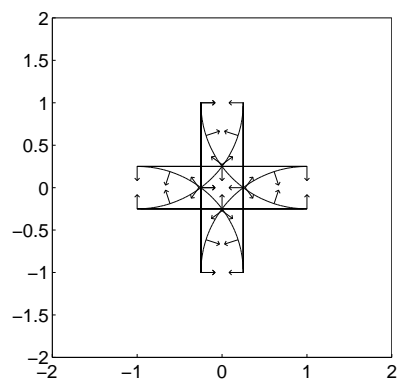

(b)

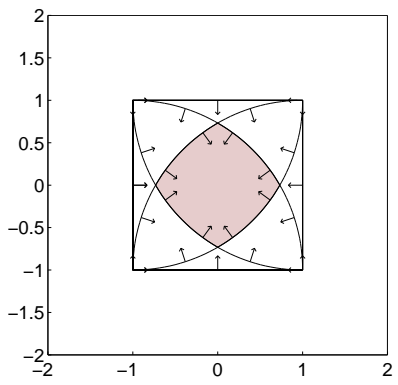

(c)

Figure 10: Visualization of directed set for Example 4.7 for (a) $r=0.5$, (b) $r=1.25$, (c) $r=2.0$

The operations of addition and scalar multiplication commute with the embedding and the visualization, cf. [3]. Namely, for $A, B \in \mathcal{C}\left(\mathbb{R}^{n}\right)$ and $\lambda \geq 0$ :

$$
\begin{array}{c|c}
J_{n}(A)+J_{n}(B)=J_{n}(A+B), & V_{n}(\vec{A}+\vec{B})=A+B, \\
\lambda \cdot J_{n}(A)=J_{n}(\lambda \cdot A), & V_{n}(\lambda \cdot \vec{A})=\lambda \cdot A .
\end{array}
$$

A simple example shows that the visualization of the sum may be different from the sum of the visualizations:

$$
\begin{aligned}
0_{\mathcal{D}(\mathbb{R})} & =\overrightarrow{[-2,3]}+\overrightarrow{[2,-3]} \\
V_{1}(\overrightarrow{[-2,3]}) & =[-2,3], \quad V_{1}(\overrightarrow{[2,-3]})=\ominus V_{1}(\overrightarrow{[-2,3]})=[-3,2] \\
\{0\}=V_{1}\left(0_{\mathcal{D}(\mathbb{R})}\right) & \neq[-5,5]=[-2,3]+[-3,2]
\end{aligned}
$$

\subsection{The directed and Rubinov subdifferentials}

We are now ready to define the directed subdifferential for QD functions. 
Definition 4.8. Let $f: \mathbb{R}^{n} \rightarrow \mathbb{R}$ be quasidifferentiable. The directed subdifferential of $f$ at $x$ is defined by

$$
\vec{\partial} f(x)=J_{n}(\underline{\partial} f(x))-J_{n}(\ominus \bar{\partial} f(x)) .
$$

We call its visualization the Rubinov subdifferential:

$$
\partial_{R} f(x)=V_{n}(\vec{\partial} f(x))
$$

The vector $s \in \partial_{R} f(x)$ is called Rubinov subgradient of $f$ at $x$.

Thus, every pair of sets $[\underline{\partial} f(x), \ominus \bar{\partial} f(x)]$ determines a directed subdifferential which is an element of the Banach space of directed sets, and its visualization, a possibly non-convex set in $\mathbb{R}^{n}$.

Also, the corresponding Rubinov subdifferential is always a nonempty compact, not necessarily convex set in $\mathbb{R}^{n}[3]$.

Note that the pair of convex compacts $[\underline{\partial} f(x), \ominus \bar{\partial} f(x)]$ corresponding to the quasidifferentiable function $f$ is not unique. Actually, every equivalent to it pair in the sense of $[22,20]$ may replace it in the directional derivative. That is why, the quasidifferential of Demyanov and Rubinov [7] is not just one pair, but the whole equivalence class of pairs of convex compacts determined by this pair (see $[22,20]$ ). It is hence reasonable to check that the directed and Rubinov subdifferentials are well-defined, i.e. do not depend on the chosen pair representing the equivalence class.

Lemma 4.9. The directed and Rubinov subdifferentials are well-defined for $Q D$ functions.

Proof. We have to show that $\partial_{R} f(x)$ does not depend on the specific (equivalent) pair of sets $[U, V]=[\underline{\partial} f(x), \bar{\partial} f(x)]$.

Indeed, assume that the two equivalent pairs $[U, V]$ and $[\tilde{U}, \tilde{V}]$ satisfy

$$
\begin{aligned}
f^{\prime}(x ; \ell) & =\max _{u \in U}\langle\ell, u\rangle-\max _{v \in \ominus V}\langle\ell, v\rangle \\
& =\max _{\tilde{u} \in \tilde{U}}\langle\ell, \tilde{u}\rangle-\max _{\tilde{v} \in \odot \tilde{V}}\langle\ell, \tilde{v}\rangle .
\end{aligned}
$$

Hence,

$$
\delta^{*}(\ell, U)+\delta^{*}(\ell, \ominus \widetilde{V})=\delta^{*}(\ell, \widetilde{U})+\delta^{*}(\ell, \ominus V)
$$


By the property (1) of the support function and the property (11) of the embedding $J_{n}$

$$
\begin{aligned}
U+\ominus \widetilde{V} & =\widetilde{U}+\ominus V, \\
J_{n}(U)+J_{n}(\ominus \widetilde{V}) & =J_{n}(\widetilde{U})+J_{n}(\ominus V), \\
J_{n}(U)-J_{n}(\ominus V) & =J_{n}(\widetilde{U})-J_{n}(\ominus V) .
\end{aligned}
$$

Thus, the directed subdifferential, and hence the Rubinov one, do not depend on the representing pair.

We also note that the above definition of directed and Rubinov subdifferentials extends the corresponding definition from the class of DC functions $[4,6]$ to the class of QD functions. For DC functions these definitions coincide.

Lemma 4.10. If $f: \mathbb{R}^{n} \rightarrow \mathbb{R}$ is DC with $f=g-h$, $g$, h are convex, then $f$ is $Q D$ and

$$
\begin{aligned}
f^{\prime}(x ; l) & =g^{\prime}(x ; l)-h^{\prime}(x ; l), \\
\mathcal{D} f(x) & =[\partial g(x), \ominus \partial h(x)], \\
\vec{\partial} f(x) & =J_{n}(\partial g(x))-J_{n}(\partial h(x)) .
\end{aligned}
$$

Proof. A DC function is directionally differentiable, since the directional derivatives of $g$ and $h$ exist in $x$ from $l$, i.e.

$$
f^{\prime}(x ; l)=g^{\prime}(x ; l)-h^{\prime}(x ; l) .
$$

The directional derivative of a convex function is the support function of the Moreau-Rockafellar subdifferential, i.e.

$$
f^{\prime}(x ; l)=\delta^{*}(l, \partial g(x))-\delta^{*}(l, \partial h(x))=\max _{u \in \partial g(x)}\langle l, u\rangle+\min _{v \in \ominus \partial h(x)}\langle l, v\rangle
$$

and $\mathcal{D} f(x)=[\partial g(x), \ominus \partial h(x)]$.

The definition of the directed subdifferential yields

$$
\vec{\partial} f(x)=J_{n}(\partial g(x))-J_{n}(\ominus(\ominus \partial h(x)))=J_{n}(\partial g(x))-J_{n}(\partial h(x)) .
$$




\section{Directed subdifferential for lower- $\mathrm{C}^{k}$ and amenable functions}

In $[23,(1.6)]$ and $[24$, Definition 10.29] (as one example of "subsmooth" functions), the class of lower- $\mathrm{C}^{k}$ functions is introduced. Upper- $\mathrm{C}^{k}$ functions can be defined in a symmetric way. As it is shown in this section, these classes constitute important subclasses of the class of QD functions.

Definition 5.1. The function $f: \mathbb{R}^{n} \rightarrow \mathbb{R}$ is called lower- $\mathrm{C}^{k}$ with $k \in$ $\mathbb{N} \cup\{\infty\}$, if it has the form

$$
f(x)=\sup _{p \in P} F(x, p) \quad\left(x \in \mathbb{R}^{n}\right),
$$

where $P$ is a compact topological space and $F: \mathbb{R}^{n} \times P \rightarrow \mathbb{R}$ is a function that has partial derivatives w.r.t. $x$ up to order $k$ with both $F$ and these derivatives (jointly) continuous w.r.t. $(x, p)$.

Hence, we may replace the supremum by a maximum.

$f: \mathbb{R}^{n} \rightarrow \mathbb{R}$ is called upper- $\mathrm{C}^{k}$, if it has the form

$$
f(x)=\inf _{p \in P} F(x, p) \quad\left(x \in \mathbb{R}^{n}\right) .
$$

Obviously, $f$ is upper- $\mathrm{C}^{k}$ if and only if $-f$ is lower- $\mathrm{C}^{k}, k \in \mathbb{N} \cup\{\infty\}$. A characterization of lower- $\mathrm{C}^{2}$ functions is stated in [23, Theorem 6].

Proposition 5.2. Let $f: \mathbb{R}^{n} \rightarrow \mathbb{R}$ be locally Lipschitz.

Then, $f$ is lower- $C^{2}$ if and only if there exists a convex neighborhood of each point $x \in \mathbb{R}^{n}$ such that $f=g-h$ is $D C$ with $g, h$ convex and $h$ is additionally a quadratic function.

The following proposition calculates the directed subdifferential for a wider class of functions than the one of lower- $\mathrm{C}^{2}$ functions.

Proposition 5.3. Let $f: \mathbb{R}^{n} \rightarrow \mathbb{R}$ be in the form

$$
f(x)=g(x)-h(x)
$$

with $g$ quasidifferentiable and $h \in C^{1}\left(\mathbb{R}^{n}\right)$.

Then

(i) $f$ is quasidifferentiable with $f^{\prime}(x ; \ell)=g^{\prime}(x ; \ell)-\nabla h(x) \ell$; 
(ii) $\vec{\partial} f(x)=\vec{\partial} g(x)-J_{n}\left(\left\{\nabla h(x)^{\top}\right\}\right)$.

Proof. The function $h$ is obviously directionally differentiable with $h^{\prime}(x ; \ell)$ $=\nabla h(x) \ell$. Hence, the quasidifferentiability of $g$ yields

$$
\begin{aligned}
f^{\prime}(x ; \ell) & =g^{\prime}(x ; \ell)-\nabla h(x) \ell \\
& =\delta^{*}\left(\ell, \underline{\partial} g(x)+\left\{-\nabla h(x)^{\top}\right\}\right)-\delta^{*}(\ell, \ominus \bar{\partial} g(x)) .
\end{aligned}
$$

Hence, $f$ is quasidifferentiable with

$$
\begin{aligned}
\vec{\partial} f(x) & =J_{n}\left(\underline{\partial} g(x)+\left\{-\nabla h(x)^{\top}\right\}\right)-J_{n}(\ominus \bar{\partial} g(x)) \\
& =\vec{\partial} g(x)-J_{n}\left(\left\{\nabla h(x)^{\top}\right\}\right) .
\end{aligned}
$$

Clearly, the previous proposition can also be applied for lower- $\mathrm{C}^{2}$ functions.

Corollary 5.4. Let $f: \mathbb{R}^{n} \rightarrow \mathbb{R}$ be lower- $C^{k}$ with $k \in \mathbb{N} \cup\{\infty\}, k \geq 2$ and $f=g-h, h \in C^{k}$.

Then $f$ is quasidifferentiable and

$$
\vec{\partial} f(x)=J_{n}(\partial g(x))-J_{n}\left(\left\{\nabla h(x)^{\top}\right\}\right) .
$$

If $\tilde{f}$ is upper- $C^{k}$ and $-\tilde{f}=g-h$, then $\tilde{f}$ is also quasidifferentiable with

$$
\vec{\partial} \widetilde{f}(x)=-J_{n}(\partial g(x))+J_{n}\left(\left\{\nabla h(x)^{\top}\right\}\right) .
$$

Proof. The proof follows easily from Proposition 5.3, since each convex function $g$ is DC and hence QD.

The formula for upper- $\mathrm{C}^{k}$ functions follows immediately from

$$
\vec{\partial} \widetilde{f}(x)=-\vec{\partial}(-\widetilde{f})(x)=-\vec{\partial}(g-h)(x) .
$$

Let us now consider the wider class of lower- $\mathrm{C}^{1}$ functions. Since a DC representation for lower- $\mathrm{C}^{1}$ functions might not exist similarly to Proposition 5.2, we state a possibility to calculate the directed subdifferential for this specific class of quasidifferentiable functions.

Proposition 5.5. Let $f: \mathbb{R}^{n} \rightarrow \mathbb{R}$ be lower- $C^{1}$, i.e.

$$
f(x)=\max _{p \in P} F(x, p) \quad\left(x \in \mathbb{R}^{n}\right)
$$

with $\nabla_{x} F$ being (jointly) continuous w.r.t. $(x, p)$. Then, 
(i) the function $f$ is quasidifferentiable with $f^{\prime}(x ; \ell)=\sup _{p \in I_{P}(x)} F^{\prime}(x ; p ; \ell)$, where $I_{P}(x)=\{p \in P \mid F(x, p)=f(x)\}$ is the set of active indices, and $F^{\prime}(x ; p ; \ell)$ is the directional derivative of $F$ w.r.t. $x$ at $(x, p)$ in the direction $l$.

(ii) $\vec{\partial} f(x)=J_{n}\left(\operatorname{co}\left(\bigcup_{p \in I_{P}(x)}\left\{\nabla_{x} F(x, p)\right\}\right)\right)$.

Proof. The formula for the directional derivative can be found in [23, $(2.1)-$ $(2.2)]$. Since $F$ is continuously differentiable w.r.t. $x$, we have $F_{x}^{\prime}(x ; p ; \ell)=$ $\nabla_{x} F(x, p) l$. The supremum in the expression (4) for directional derivative is thus equivalent to the support function of the convex hull of the active gradients (observe that the convex hull is closed, as the set $I_{P}(x)$ is compact and the gradients depend continuously on the parameter $p$ ). As a consequence, $f$ is $\mathrm{QD}$ and the formula for the directed subdifferential follows from the definition.

The following definition of amenable functions is taken from [24, Definition 10.23] and [17, Sec. 3.2, remarks before Corollary 3.76]. Amenable functions are another important subclass of QD functions.

Definition 5.6. The function $f: \mathbb{R}^{n} \rightarrow \mathbb{R} \cup\{ \pm \infty\}$ is called amenable, if it has the form $f=g \circ \varphi$ with $g: \mathbb{R}^{m} \rightarrow \mathbb{R} \cup\{ \pm \infty\}$ proper, l.s.c., convex and $\varphi \in C^{1}\left(\mathbb{R}^{n} ; \mathbb{R}^{m}\right)$ such that the following constraint qualification holds. Whenever we have the equation

$$
J \varphi(x)^{\top} y=0 \quad \text { for some } y \in N_{D}(\varphi(x)),
$$

in a neighborhood of $x$, then this vector $y$ must be zero.

Here, $J \varphi(x)$ denotes the Jacobian of $\varphi$ at $x$ and $N_{D}(z)$ is the normal cone (see [24, Definition 6.3]) to the closure of the effective domain of $g: D=$ $\operatorname{cl}(\operatorname{dom}(g))$ at $z=\varphi(x)$.

An amenable function $f: \mathbb{R}^{n} \rightarrow \mathbb{R}$ is called strongly amenable, if $\varphi \in C^{2}$.

As we do not consider functions with $\operatorname{dom}(f) \neq \mathbb{R}^{n}$, the normal cone to the effective domain is a singleton $N_{D}(z)=\{0\}$, and hence the qualification condition is always satisfied.

Let us first calculate the directed subdifferential for a subclass of QD functions larger than the class of amenable functions. 
Proposition 5.7. Let $f: \mathbb{R}^{n} \rightarrow \mathbb{R}$ be in the form

$$
f(x)=(g \circ \varphi)(x)
$$

with $g$ quasidifferentiable and locally Lipschitz, $\varphi \in C^{1}$.

Then,

(i) the function $f$ is quasidifferentiable with directional derivative $f^{\prime}(x ; \ell)$ $=g^{\prime}(\varphi(x) ; J \varphi(x) \ell)$

(ii) $\vec{\partial} f(x)=J \varphi(x)^{\top} \vec{\partial} g(\varphi(x))$.

Proof. The function $f$ is directionally differentiable under the assumptions, since the $\mathrm{C}^{1}$-property of $\varphi$ and the locally Lipschitz continuity of $g$ yields

$$
\begin{aligned}
g(\varphi(x+h \ell)) & =g(\varphi(x)+h J \varphi(x) \ell+\mathcal{O}(h)) \\
& =g(\varphi(x)+h J \varphi(x) \ell)+\mathcal{O}(h) .
\end{aligned}
$$

Let us express the directional derivative of $f$ by that of $g$ :

$$
\begin{aligned}
f^{\prime}(x ; \ell) & =\lim _{h \downarrow 0} \frac{g(\varphi(x+h \ell))-g(\varphi(x))}{h} \\
& =\lim _{h \downarrow 0} \frac{g(\varphi(x)+h J \varphi(x) \ell)+\mathcal{O}(h)-g(\varphi(x))}{h} \\
& =\lim _{h \downarrow 0} \frac{g(\varphi(x)+h J \varphi(x) \ell)-g(\varphi(x))}{h}=g^{\prime}(\varphi(x) ; J \varphi(x) \ell) .
\end{aligned}
$$

Now, the quasidifferentiability of $g$ yields

$$
\begin{aligned}
f^{\prime}(x ; \ell) & =g^{\prime}(\varphi(x) ; J \varphi(x) \ell) \\
& =\delta^{*}(J \varphi(x) \ell, \underline{\partial} g(\varphi(x)))-\delta^{*}(J \varphi(x) \ell, \ominus \bar{\partial} g(\varphi(x))) \\
& =\delta^{*}\left(\ell, J \varphi(x)^{\top} \underline{\partial} g(\varphi(x))\right)-\delta^{*}\left(\ell, \ominus J \varphi(x)^{\top} \bar{\partial} g(\varphi(x))\right) .
\end{aligned}
$$

We found a representation for calculating the directed subdifferential and use the definition (7) of the linear image of a directed set, i.e. a matrix applied to a directed set:

$$
\begin{aligned}
\vec{\partial} f(x) & =J_{n}\left(J \varphi(x)^{\top} \underline{\partial} g(\varphi(x))\right)-J_{n}\left(\ominus J \varphi(x)^{\top} \bar{\partial} g(\varphi(x))\right) \\
& =J \varphi(x)^{\top}\left(J_{n}(\underline{\partial} g(\varphi(x)))-J_{n}(\ominus \bar{\partial} g(\varphi(x)))\right) \\
& =J \varphi(x)^{\top} \vec{\partial} g(\varphi(x))
\end{aligned}
$$


Trivially, we can apply the previous proposition for amenable functions.

Corollary 5.8. Let $f: \mathbb{R}^{n} \rightarrow \mathbb{R}$ be amenable in the form $f(x)=(g \circ \varphi)(x)$. Then $f$ is quasidifferentiable and

$$
\vec{\partial} f(x)=J_{n}\left(J \varphi(x)^{\top} \partial g(\varphi(x))\right)
$$

Proof. Observe that $\mathcal{D} g(y)=[\partial g(y),\{0\}]$, hence, the statement follows immediately from $g^{\prime}(x ; \ell)=\delta^{*}(\ell, \partial g(x))$ and the previous proposition:

$$
\begin{aligned}
\vec{\partial} f(x) & =J \varphi(x)^{\top} \vec{\partial} g(\varphi(x))=J \varphi(x)^{\top} J_{n}(\partial g(\varphi(x))) \\
& =J_{n}\left(J \varphi(x)^{\top} \partial g(\varphi(x))\right),
\end{aligned}
$$

where we used the definition of a linear image of a directed set.

\section{Conclusions}

In this first part we extended the notion of the directed subdifferential to the class of QD functions more general than the smaller class of DC functions. For the specific class of QD functions, the amenable and lower$\mathrm{C}^{k}$ functions we obtained exact formulas for the directed subdifferential. In the second part we will list the calculus rules which are inherited from the quasidifferential calculus. A second focus will be laid on optimality conditions and the connections to some known convex and non-convex subdifferentials.

Acknowledgements. The authors would like to thank Boris Mordukhovich who encouraged them to extend the directed subdifferential to amenable and lower- $\mathrm{C}^{k}$ functions. This work was partially supported by The Hermann Minkowski Center for Geometry at Tel Aviv University.

\section{References}

[1] R. Baier, M. Dellnitz, M. Hessel-von Molo, I. G. Kevrekidis, and S. Sertl. The computation of invariant sets via Newton's method. Submitted, 21 pages, http://num.math.uni-bayreuth.de/de/ publications/2010/baier_et_al_the_comp_of_invar_sets_2010/, May 2010.

[2] R. Baier and E. Farkhi. Differences of convex compact sets in the space of directed sets, Part I: The space of directed sets. Set-Valued Anal., 9(3):217-245, 2001. 
[3] R. Baier and E. Farkhi. Differences of convex compact sets in the space of directed sets, Part II: Visualization of directed sets. Set-Valued Anal., 9(3):247-272, 2001.

[4] R. Baier and E. Farkhi. The directed subdifferential of DC functions. In A. Leizarowitz, B. S. Mordukhovich, I. Shafrir, and A. J. Zaslavski, editors, Nonlinear Analysis and Optimization II: Optimization. A Conference in Celebration of Alex Ioffe's 70th and Simeon Reich's 60th Birthdays, June 18-24, 2008, Haifa, Israel, volume 513 of AMS Contemp. Math., pages 27-43. AMS and Bar-Ilan University, 2010.

[5] R. Baier, E. Farkhi, and V. Roshchina. The directed and Rubinov subdifferentials of quasidifferentiable functions, Part II: Calculus. Nonlinear Anal. (same volume).

[6] R. Baier, E. Farkhi, and V. Roshchina. On computing the Mordukhovich subdifferential using directed sets in two dimensions. In R. S. Burachik and Jen-Chih Yao, editors, Variational Analysis and Generalized Differentiation in Optimization and Control. In Honor of Boris S. Mordukhovich, volume 47 of Springer Optimization and Its Applications, pages 59-93. Springer, New York-Dordrecht-Heidelberg-London, 2010.

[7] V. F. Demyanov and A. M. Rubinov. Constructive Nonsmooth Analysis, volume 7 of Approximation and Optimization. Verlag Peter Lang, Frankfurt/Main, 1995. Russian original "Foundations of Nonsmooth Analysis, and Quasidifferential Calculus" published in Nauka, Moscow, 1990.

[8] P. Diamond, P. Kloeden, A. Rubinov, and A. Vladimirov. Comparative properties of three metrics in the space of compact convex sets. SetValued Anal., 5(3):267-289, 1997.

[9] H. Hadwiger. Minkowskische Addition und Subtraktion beliebiger Punktmengen und die Theoreme von Erhard Schmidt. Math. Z., 53(3):210-218, 1950.

[10] E. Hewitt and K. Stromberg. Real and Abstract Analysis, volume 25 of Graduate Texts in Mathematics. Springer-Verlag, New York, 1975. Third printing, first published in 1965. 
[11] J.-B. Hiriart-Urruty. Generalized differentiability, duality and optimization for problems dealing with differences of convex functions. In J. Ponstein, editor, Convexity and Duality in Optimization. Proceedings of the Symposium on Convexity and Duality in Optimization Held at the University of Groningen, The Netherlands, June 22, 1984, volume 256 of Lecture Notes in Econom. and Math. Systems, pages 37-70, BerlinHeidelberg-New York-Tokyo, 1985. Springer.

[12] J.-B. Hiriart-Urruty and C. Lemaréchal. Convex Analysis and Minimization Algorithms I. Fundamentals, volume 305 of Grundlehren der mathematischen Wissenschaften. Springer, Berlin-Heidelberg-New York, 1993.

[13] R. Horst, P. M. Pardalos, and N. V. Thoai. Introduction to Global Optimization, volume 48 of Nonconvex Optimization and its Applications. Kluwer Acad. Publ., Dordrecht, second edition, 2000.

[14] R. Horst and N. V. Thoai. DC programming: overview. J. Optim. Theory Appl., 103(1):1-43, 1999.

[15] E. Kaucher. Interval analysis in the extended interval space I R. In Fundamentals of Numerical Computation (Computer-Oriented Numerical Analysis) (Proc. Conf., Tech. Univ. Berlin, Berlin, 1979), volume 2 of Comput. Suppl., pages 33-49. Springer, Vienna, 1980.

[16] S. Markov. On directed interval arithmetic and its applications. J.UCS, 1(7):514-526 (electronic), 1995.

[17] B. S. Mordukhovich. Variational Analysis and Generalized Differentiation. I Basic Theory, volume 330 of Grundlehren der Mathematischen Wissenschaften. Springer-Verlag, Berlin, 2006.

[18] E. Nurminski. Subtraction of convex sets and its application in $\varepsilon$ subdifferential calculus. IIASA Working Paper WP-82-083, Internat. Inst. Appl. Systems Anal. (IIASA), Laxenburg, September 1982. 32 pp.

[19] D. Pallaschke and R. Urbański. Minimal pairs of compact convex sets, with application to quasidifferential calculus. In Quasidifferentiability and Related Topics, volume 43 of Nonconvex Optim. Appl., pages 173213. Kluwer Acad. Publ., Dordrecht, 2000. 
[20] D. Pallaschke and R. Urbański. Pairs of Compact Convex Sets. Fractional Arithmetic with Convex Sets, volume 548 of Mathematics and Its Applications. Kluwer Acad. Publ., Dordrecht, 2002.

[21] L. S. Pontryagin. Linear differential games. ii. Sov. Math., Dokl., 8(4):910-912, 1967.

[22] H. Rådström. An embedding theorem for spaces of convex sets. Proc. Amer. Math. Soc., 3:165-169, 1952.

[23] R. T. Rockafellar. Favorable classes of Lipschitz-continuous functions in subgradient optimization. In Progress in Nondifferentiable Optimization, volume 8 of IIASA Collaborative Proc. Ser. CP-82, pages 125-143. Internat. Inst. Appl. Systems Anal. (IIASA), Laxenburg, 1982.

[24] R. T. Rockafellar and R. J.-B. Wets. Variational Analysis, volume 317 of Grundlehren der Mathematischen Wissenschaften [Fundamental Principles of Mathematical Sciences]. Springer-Verlag, Berlin, 1998.

[25] A. M. Rubinov and I. S. Akhundov. Difference of compact sets in the sense of Demyanov and its application to non-smooth analysis. Optimization, 23(3):179-188, 1992.

[26] A. Shapiro. On functions representable as a difference of two convex functions in inequality constrained optimization. Research report University of South Africa, 1983.

[27] Xianfu Wang. Pathological Lipschitz Functions In $\mathbb{R}^{N}$. PhD thesis, Simon Fraser University, Department of Mathematics \& Statistics, Burnaby, B.C., Canada, June 1975. Master of Science thesis. http://ir.lib.sfu.ca/bitstream/1892/8022/1/b17501684.pdf. 